\title{
Entrelacs
}

Cinéma et audiovisuel

\section{Monde « réel » et monde imaginaire. Le rôle de la bande son dans Eraserhead, de David Lynch}

\section{Emmanuelle Bobée}

\section{(2) OpenEdition}

\section{Journals}

Édition électronique

URL : http://journals.openedition.org/entrelacs/236

DOI : 10.4000/entrelacs.236

ISSN : 2261-5482

Éditeur

Éditions Téraèdre

\section{Édition imprimée}

Date de publication : 1 février 2011

ISBN : 978-2-360850-03-7

ISSN : 1266-7188

\section{Référence électronique}

Emmanuelle Bobée, « Monde « réel » et monde imaginaire. Le rôle de la bande son dans Eraserhead, de David Lynch », Entrelacs [En ligne], 8 | 2011, mis en ligne le 01 août 2012, consulté le 19 avril 2019. URL : http://journals.openedition.org/entrelacs/236 ; DOI : 10.4000/entrelacs.236

Ce document a été généré automatiquement le 19 avril 2019

Tous droits réservés 


\title{
Monde « réel » et monde imaginaire. Le rôle de la bande son dans Eraserhead, de David Lynch
}

\author{
Emmanuelle Bobée
}

1 Au cinéma comme en littérature, toute œuvre de fiction repose sur un accord tacite selon lequel le spectateur ou le lecteur accepte de considérer temporairement les événements représentés comme "réels» - ce que Samuel Taylor Coleridge nomme la «suspension volontaire d'incrédulité » (willing suspension of disbelief). Ainsi, « le spectateur acceptera [...] des postulats narratifs qui seraient impossibles dans le monde réel, et considérera les événements narrés non pas selon leur véracité, mais selon leur vraisemblance dans le cadre du monde postulé par le genre. $»^{1}$ Tandis que le roman nécessite une reconstruction mentale de l'univers fictionnel par le lecteur, le dispositif cinématographique permet, grâce à ses spécificités (image mouvante, dialogues et sons ambiants), de donner au film de fiction l'apparence (illusoire) de la réalité.

2 Toutefois, les mécanismes perceptifs mis en jeu par le spectateur dans la réception d'une œuvre audiovisuelle diffèrent sensiblement de ceux qui opèrent dans la vie courante. En effet, tandis que les images qui nous parviennent du monde extérieur proviennent de notre propre foyer perceptif, en l'occurrence une paire d'yeux raccordée à un cortex visuel situé en un point précis de l'espace, les images filmiques sont médiatisées par l'objectif de la caméra et, au-delà, par le réalisateur qui positionne celle-ci dans le décor. Au fil des prises successives d'une même scène, la caméra est susceptible d'être déplacée afin de modifier le point de vue sur l'action - la scène peut également être filmée simultanément par plusieurs appareils -, ce qui permet, gr âce au montage des prises de vue, de montrer des événements fictifs sous différents angles. Tandis que nous sommes dans l'impossibilité de nous trouver simultanément en plusieurs points de l'espace, la magie du cinéma permet ce dédoublement, cette démultiplication des points de vue.

3 Au fur et à mesure de l'évolution des techniques cinématographiques, les spectateurs ont intégré de nouveaux codes narratifs et se sont progressivement adaptés à une situation qui, sur le plan perceptif, n'est rien moins que naturelle. Dans le même ordre d'idée, nous 
ne sommes guère habitués à entendre les pensées de nos contemporains ou à visualiser leurs fantasmes, rêves et autres souvenirs. Or ce procédé est devenu monnaie courante au cinéma : des scènes rêvées, fantasmées ou remémor ées par un personnage peuvent faire partie intégrante du récit cinématographique, au même titre que les scènes « réelles ». Le personnage " "s'empare" d'une partie de la narration du film » et accorde au spectateur "le privilège de lire dans ses pensées $~^{2}$. Ainsi se dévoile un autre monde fictif, un monde méta-diégétique, situé à la fois dans et au-delà de la diégèse (d'où l'utilisation du préfixe grec méta- qui signifie « après » ou « au-delà de »).

Dès lors, comment le spectateur peut-il faire la distinction entre ce qui est « réel », du point de vue des personnages, et ce qui ne l'est pas? Si les cinéastes disposent, à cet effet, d'un large éventail de procédés visuels (par exemple, un gros plan sur le visage du personnage rêvant ou se souvenant, suivi d'un fondu enchaîné sur la scène rêvée ou remémorée), la bande son - trop souvent, hélas, cantonnée à des fonctions purement illustratives - constitue parfois un é lément primordial du récit cinématographique. Certains sons peuvent être envisagés comme la manifestation d'une subjectivité interne au récit, qui donne au spectateur la possibilité d'entrer en communication directe avec les personnages, leur perception du monde diégétique qui les entoure ou leur monde imaginaire. En nous appuyant sur les recherches menées, depuis un demi-siècle, par de nombreux théoriciens du récit littéraire et cinématographique, nous proposerons une classification des sons filmiques permettant de délimiter les espaces fictionnels réel et imaginaire. Nous illustrerons ensuite notre propos par une étude du traitement sonore de quelques scènes d'Eraserhead, le premier long métrage de David Lynch.

En premier lieu, il convient d'opérer la distinction entre le récit focalisé ${ }^{3}$, qui reflète la perception du monde diégétique par un personnage (le spectateur voit/entend ce que ce dernier est censé voir/entendre), et l'énonciation d'un monde méta-diégétique, ou monde imaginaire, par une instance énonciative secondaire ${ }^{4}$, dont la subjectivité se manifeste de façon plus ou moins apparente par des "marques énonciatives » visuelles (gros plan sur le visage du personnage, fondu enchaîné, utilisation du flou ou du ralenti, passage au noir et blanc) ou auditives (augmentation ou diminution du volume sonore, déformation du son par l'ajout d'effets tels que la réverbération ou l'écho). Sur le plan sonore, Edward Branigan distingue le " point de vue auriculaire » (« aural point of view») et la "perception auriculaire » (« aural perception»), selon que «nous entendons depuis le point de l'espace où se trouve le personnage » ou que « nous entendons ce que le personnage seul entend $»^{5}$ . Cette approche présente l'avantage de poser clairement la différence entre les sons diégétiques réels qui font l'objet d'une perception différenciée et les sons purement imaginaires (sons rêvés, remémorés ou fantasmés).

6 La distinction entre les sons diégétiques réels - qu'ils soient subjectifs ou non - et les sons imaginaires repose sur l'identification de la source sonore, selon qu'elle est localisée ou non dans l'espace diégétique premier. Toute la difficulté tient au fait que le son peut provenir d'une source diégétique non visualisée (cas des sons «hors champ»), voire d'une source non diégétique. La prise en compte du contexte narratif s'avère donc indispensable pour dé terminer la nature réelle ou imaginaire, diégétique ou non diégétique d'un son donné. Par ailleurs, le caractère objectif ou subjectif d'un son diégétique réel renvoie à la notion de point d'écoute, que Michel Chion définit de la manière suivante : «Dans une séquence audiovisuelle cette notion [...] désigne: 1) Le point à partir duquel il nous semble pouvoir dire que nous entendons un son comme proche ou lointain de nous, point qui soit concorde avec la place de la caméra, soit en est 
différent (cas fréquent du personnage éloigné dans l'image et de sa voix entendue proche). C'est alors le point d'écoute au sens spatial. 2) Le personnage par les oreilles duquel il est suggéré que nous entendons un son (par exemple, s'il parle au téléphone, et que nous entendons distinctement son interlocuteur comme lui-même est censé l'entendre). C'est alors le point d'écoute au sens subjectif. ${ }^{6}$ Dans un souci d'uniformité, nous adopterons l'acception spatiale du terme, en y englobant la conception subjective : le point d'écoute est situé en un point relativement précis de l'espace diégétique, y compris, le cas échéant, dans la tête de l'un des personnages.

7 La classification que nous proposons tient compte à la fois de la localisation de la source sonore (émission du son) et de l'emplacement du point d'écoute (réception du son). Examinons les différents cas de figure qui peuvent se présenter lorsqu'un son (parole, musique, bruit) se fait entendre dans une scène comportant à l'écran deux personnages $\mathrm{A}$ et $\mathrm{B}$.

$\left.81^{\circ}\right)$ La source sonore se trouve dans le champ et le point d'écoute es t objectif, c'est-à-dire à la place de la caméra, coïncidant avec le point de vue. L'origine du son est identifiée et les personnages $\mathrm{A}$ et $\mathrm{B}$ entendent $a$ priori la même chose : il s'agit d'un son réel objectif que nous nommerons « in ", en référence à la terminologie de Michel Chion ${ }^{7}$. On peut distinguer celui dont la source est visualisée et celui dont la source est dans le champ, mais non visualisée (personnage filmé de dos en train de parler, source sonore masquée par un obstacle, etc.).

$92^{\circ}$ ) La source sonore n'est pas située dans le champ, mais est clairement identifiée comme appartenant à l'espace diégétique, soit parce qu'elle a été visualisée auparavant, soit parce que sa présence découle naturellement de l'action montrée à l'écran ${ }^{8}$. Le point d'écoute est «objectif» et les personnages $A$ et $B$ entendent la même chose: il s'agit également d'un son réel objectif, cette fois qualifié de « hors-champ ».

$103^{\circ}$ ) La source sonore est dans le champ ou hors-champ et le point d'écoute est " subjectif ", c'est-à-dire que le spectateur a une perception auditive identique à celle du personnage focalisateur A, mais différente de celle du personnage B : il s'agit d'un son réel subjectif. Lorsque la perception auditive du personnage A est modifiée par des facteurs physiques (perte d'audition) ou émotionnels (fatigue extrême, état de choc), on parle de "son (réel) subjectif déformé ». Cela se manifeste généralement soit par une baisse ou une augmentation de l'intensité sonore ${ }^{9}$, soit par l'ajout de filtres, de réverbération ou d'écho ${ }^{10}$. Le caractère subjectif du son peut être lié à la proximité de la source sonore, indépendamment des facultés mentales ou auditives du personnagerécepteur: les personnages $\mathrm{A}$ et $\mathrm{B}$ sont interchangeables. On peut donc le qualifier de "son (réel) subjectif externe». Le cas le plus évident est celui du téléphone (seul le personnage A entend la voix de son interlocuteur), mais on peut également mentionner le cas d'un personnage muni d'écouteurs stéréophoniques ou d'un « kit mains libres ». Si la source sonore se trouve dans le corps du personnage A (respiration, battements de cœur), celui-ci est nécessairement amené à l'entendre avec une acuité particulière. On parlera donc de « son (réel) subjectif interne ».

$114^{\circ}$ ) La source sonor e ne se trouve pas à proprement parler dans l'espace diégétique premier, mais dans la tête du personnage $\mathrm{A}$; le point d'écoute est donc nécessairement subjectif. Il s'agit d'un son imaginaire qui, bien qu'il n'existe pas "physiquement», est entendu par le personnage comme s'il était réel. On trouve dans cette catégorie les sons rêvés, fantasmés ou hallucinés, qui peuvent soit être purement « inventés », soit avoir été réels à un moment donné (son remémoré) ou être susceptibles de le devenir (son anticipé 
$\left.{ }^{11}\right)$, mais aussi la voix intérieure. Dans la mesure où le personnage se recentre vers son propre monde intérieur, le son imaginaire peut être accompagné d'une diminution ou d'une suppression des sons diégétiques environnants.

$5^{\circ}$ ) Le son non diégétique se définit par élimination lorsque la source sonore n'est pas visible dans le champ, ne peut pas être présumée hors-champ et ne répond pas aux caractéristiques du son imaginaire tel qu'il a été défini précédemment : la source est donc nécessairement située à l'extérieur de l'espace diégétique (les personnages $\mathrm{A}$ et $\mathrm{B}$ ne l'entendent pas). Peuvent figurer dans cette catégorie les musiques originales spécia lement créées pour le film et les musiques empruntées, les ponctuations destinées à accentuer l'effet comique ou dramatique et certaines ambiances sonores.

Cf. Tableau page ci-contre :

Proposition de classification des sons filmiques

\begin{tabular}{|c|c|c|c|c|}
\hline \multirow{10}{*}{ SON DIÉGÉTIQUE } & \multirow{6}{*}{ SON RÉEL } & \multirow{3}{*}{ Son réel objectif } & & $\begin{array}{l}\text { visualisé } \\
\text { clpadfb3 }\end{array}$ \\
\hline & & & & non visualisé \\
\hline & & & hor & rs-champ \\
\hline & & \multirow{3}{*}{ Son réel subjectif } & ext & erne \\
\hline & & & inte & erne \\
\hline & & & déf & Formé \\
\hline & \multirow{4}{*}{ SON IMAGINAIRE } & \multicolumn{3}{|c|}{ Son rêvé ou halluciné } \\
\hline & & \multicolumn{3}{|c|}{ Son remémoré ou anticipé } \\
\hline & & \multicolumn{3}{|l|}{ Voix intérieure } \\
\hline & & \multicolumn{3}{|l|}{ Lecture d'un texte } \\
\hline
\end{tabular}

14 À partir de cette classification, on peut mettre en évidence les différents niveaux énonciatifs (extradiégétique, diégétique, méta-diégétique) et opérer la distinction entre l'énonciation principale, l'énonciation principale avec focalisation sur un personnage et l'énonciation secondaire :

- son réel objectif $\rightarrow$ énonciation principale ;

- son réel subjectif $\rightarrow$ énonciation principale avec focalisation auditive ;

- son imaginaire $\rightarrow$ énonciation secondaire.

Examinons à présent le traitement sonore de quelques scènes d'Eraserhead (David Lynch, 1977), dans lesquelles on observe une perception subjective du réel par le personnage principal et/ou la création d'un monde imaginaire. 
16 La première scène étudiée se déroule peu après le début du film (13'12 - 14'26). Après avoir parcouru un terrain vague et longé quelques pâtés d'usines dans une cité industrielle sinistre et désaffectée, Henry rentre chez lui - une chambre pauvrement meublée -, fait jouer un disque sur l'électrophone (son diégétique réel, dont la source est visualisée), s'assoit sur le lit en face du radiateur, puis semble se perdre dans ses pensées. Outre la musique, qui s'accompagne de craquements caractéristiques de l'aiguille sur le sillon, on perçoit nettement un sifflement continu, dont l'origine nous est, pour le moment, inconnue ; il pourrait s'agir d'un son hors champ (une bouilloire ?) ou d'un son non diégétique. À mesure que la caméra se rapproche du visage pensif d'Henry, l'intensité du sifflement s'accroît, tandis que la musique s'efface progressivement. Le plan suivant nous montre le radiateur, dont le robinet laisse échapper un filet de vapeur continu. Nous comprenons alors que le sifflement entendu depuis qu'Henry est entré dans la pièce provient de cette source sonore (son diégétique réel hors champ, puis in). L'augmentation de l'intensité du sifflement, qui s'accompagne d'une diminution du volume sonore de la musique, traduit une perception subjective du réel par le héros, dont l'attention est focalisée par l'objet-source sonore : le radiateur. Le dispositif sonore est complété par l'apparition de nappes sonores, dont l'origine est manifestement extra-diégétique ou méta-diégétique (il pourrait s'agir d'un "son subconscient ", catégorie intermédiaire entre le son imaginaire et le son non diégétique qui ne figure pas dans notre classification, censé traduire à la fois l'état de rêverie dans lequel est plongé le personnage et le caractère surnaturel des événements présentés). Lorsque la caméra revient sur Henry, des b ruits de l'extérieur nous parviennent amplifiés par un écho (focalisation auditive), puis les nappes disparaissent et le sifflement diminue à mesure qu'il recouvre ses esprits. La musique s'est arrêtée, mais le bruit que produit l'aiguille sur le dernier sillon nous indique un retour à la réalité « objective ».

Outre le fait qu'elle reflète une perception déformée du réel, la suppression des bruits diégétiques environnants est parfois utilisée pour créer un climat onirique. Mladen Milicevic effectue un parallèle intéressant entre les états "hypnagogique " (phase d'endormissement) et "hypnopompique » (phase précédant le réveil), et le mixage des éléments sonores : la disparition progressive des sons diégétiques, couplée à l' apparition d'une musique non diégétique, évoquerait l'entrée progressive dans le sommeil, suggérant une intériorisation, une subjectivisation des événements qui se déroulent à l'écran. Inversement, la suppression de la musique non diégétique et la réapparition concomitante des sons diégétiques marquerait un retour à la « réalité » objective du film. Ici, les bruits diégétiques environnants font l'objet d'un traitement différencié, selon qu'ils proviennent du radiateur ou du reste de la pièce. Le monde diégétique apparaît divisé entre un espace neutre et un objet particulier qui suscite un état de rêverie chez le personnage. Les sons en provenance du radiateur remplissent donc la fonction dévolue à la musique non diégétique dans le processu s décrit ci-dessus.

Dans la deuxième scène que nous nous proposons de commenter (49'10 - 52'18), Henry est allongé sur son lit et ne parvient pas à s'endormir. Le sifflement du radiateur est presque couvert par le bruit du vent qui souffle à l'extérieur (son diégétique hors champ). Soudain, le rapport s'inverse, tandis qu'un plan subjectif nous montre le radiateur filmé de côté, depuis le point de vue du personnage (focalisation visuelle et auditive). La transition entre la perception «objective » et la perception déformée de la réalité par le héros s'opère d'une manière identique à celle de la première scène étudiée, à ceci près que le monde réel est représenté, sur le plan sonore, par le souffle du vent. Une lumière 
apparaît au centre du rad iateur (apparition ponctuée par celle des nappes sonores), où l'on découvre une cavité filmée en gros plan. Dans le plan suivant, une lourde porte s'ouvre bruyamment sur un écran noir qui découvre une scène de théâtre, sur laquelle se tient une jeune femme aux joues énormes. Le sifflement et les nappes disparaissent au profit d'une musique de jazz, au rythme de laquelle la jeune femme se livre à une chorégraphie timide et cocasse. Ici, le spectateur est en droit de se demander si le monde représenté est issu de l'imaginaire d'Henry - probablement en train de rêver, cette fois ou s'il existe bel et bien, dans la réalité diégétique, un univers parallèle coincé entre le mur et le radiateur (cf. la suspension volontaire d'incrédulité, mentionnée plus haut). La disparition du son subjectif (sifflement a mplifié du radiateur) et des nappes sonores s'accompagne non seulement de l'apparition d'une musique de scène, mais également d'un retour du son diégétique ambiant (le souffle du vent), ce qui semble indiquer un effet hypnopompique, c'est-à-dire un retour à la « réalité ». D'autre part, la musique ne fait l'objet d'aucun traitement particulier de type écho ou réverbération, pouvant laisser penser qu'il s'agit d'un son imaginaire. Le seul véritable indice dont dispose le spectateur se trouve, non dans le traitement sonore de la musique, mais dans la musique elle-même. En effet, un court extrait de ce morceau a été entendu dans la première scène décrite, lorsqu'Henry déplace l'aiguille de l'électrophone en plusieurs endroits du disque. Il apparaît donc fort probable qu'il s'agisse d'un son remémoré, c'est-à-dire d'un son imaginaire, suggérant l'énonciation secondaire d'un monde enchâssé par le héros. Cette hypothèse est confortée par la manière dont se termine la scène: la musique s'efface, submergée par un souffle d'origine non diégétique (marque énonciative sonore), tandis que la jeune femme disparait dans un fondu au noir (marque visuelle).

L'ouverture en fondu (au noir) de la troisième scène choisie (1:02'55 - $1: 06$ '09) instaure une parfaite symétrie avec la fin de la scène que nous venons d'analyser. L'apparition de la « Dame du radiateur ", dans une posture rigoureusement identique (sourire aux lèvres et mains jointes), indique sans ambigüité que le rêve d' Henry reprend exactement là où il s'était arrêté. Un climat onirique s'instaure dès les premières mesures, lorsque la jeune femme entonne d'une voix presque enfantine : «In Heaven, everything is fine (ter) / You got your good things and I've got mine.» On ne décèle aucun traitement sonore particulier, à l'exception d'une légère réverbération, mais le caractère imaginaire de la scène se manifeste par une légère surexposition de l'image. Au terme de cet intermède musical, dont le message semble s'adresser autant au spectateur qu'à Henry, celui-ci rejoint timidement la jeune femme sur la scène, tandis que s'élève un souffle puissant vraisemblablement celui du vent dans la chambre d'Henry qui vient «contaminer » l'espace imaginaire. La renc ontre (précédant la fusion ultime au terme du film) de ces deux personnages et, au-delà, des espaces diégétiques réel et imaginaire, ne peut que nous laisser perplexes. En effet, si Henry assiste à des événements extraordinaires (l'apparition d'une lumière aveuglante au simple contact de la Dame du radiateur, l'évaporation soudaine de celle-ci, remplacée par un homme au visage couvert de cicatrices aperçu dans le prologue), il ne possède lui-même aucun pouvoir, aucune aptitude particulière pouvant lui être conférée par le rêve et se comporte comme un spectateur passif. La dimension "réelle " que nous lui accordons s'oppose au caractère manifestement irréel de la scène, suggérant l'idée qu'Henry se retrouve emprisonné dans son propre rêve, sans pouvoir en influencer le cours. L'environnement sonore apparait lui-même divisé en deux plans distincts : d'une part, le souffle du vent, qui évoque le monde réel - et, plus précisément, le monde extérieur, avec la notion de menace et d'imprévisibilité que peuvent porter en eux les événements climatiques -, et d'autre part 
les ponctuations sonores (nappes coïncidant avec la lumière blanche), qui soulignent le caractère irréel ou subjectif des événements montrés. Il n'est guère étonnant que le spectateur épr ouve un certain malaise, se trouvant lui-même au cœur de la dichotomie entre réalité et fiction.

Les trois scènes présentées ici mettent en évidence la construction d'un monde imaginaire, ou monde méta-diégétique, par le personnage principal du film. Nous avons souligné l'importance de la bande son qui, faut-il le rappeler, occupe une place essentielle dans le cinéma de David Lynch : «On peut dire de Lynch qu'il a rénové le cinéma par le son: si son découpage visuel est classique et transparent [...] son découpage sonore est d'emblée personnel », écrit Michel Chion. "Le son a une fonction précise, de nous propulser dans le film, de nous faire nous sentir à l'intérieur, enveloppé par sa durée. $»^{12}$ $\mathrm{Si}$, aux dires du réalisateur, le son représente la moitié du film ${ }^{13}$, l'évocation de la subjectivité du héros s'opère majoritairement par le traitement sonore et musical. L'amplification de certains sons d'origine diégétique, au détriment des bruits environnants trahit une perception subjective de la réalité diégétique, ce qui génère, la plupart du temps, un sentiment d'empathie à l'égard du personnage focalisateur - le spectateur éprouve, sur le plan auditif, ce qu'éprouve le personnage. En outre, ces marques de subjectivité laissent une porte ouverte (littéralement) sur l'imaginaire du personnage; autrement dit, la focalisation interne ouvre la voie à l'énonciation secondaire d'un monde enchâssé.

Bien que les notions de focalisation et d'énonciation soient clairement di stinctes l'énonciation relève de la voix (qui parle ?), tandis que la focalisation exprime un point de vue ou un point d'écoute (qui perçoit?) -, le traitement sonore de l'énonciation secondaire et du discours focalisé peut être identique : écho, réverbération, ajout de filtres, baisse ou augmentation du volume sonore. Lorsqu'un son a subi de telles altérations, c'est la localisation de la source sonore dans l'espace diégétique premier qui permet de déterminer si l'on est en présence d'un son subjectif (déformé) ou d'un son imaginaire. Toutefois, nous avons vu qu'un son imaginaire peut fort bien n'avoir subi aucun traitement particulier (cf. l'analyse de la troisième scène). En outre, les sons imaginaires peuvent cœxister avec les bruits diégétiques env ironnants. Dans la mesure où le personnage énonciateur d'un monde méta-diégétique se trouve, dans le même temps, au sein de l'espace diégétique premier, il est théoriquement apte à en percevoir certaines occurrences sonores et à les intégrer dans son discours, de la même façon que nous intégrons parfois à notre rêve la sonnerie (bien réelle) du téléphone, lorsque nous dormons profondément. Ce son est alors focalisé, ce qui peut donner lieu à une amplification ou une diminution du volume sonore (cf. le souffle du vent dans les deuxième et troisième scènes).

Le monde "réel » et le monde imaginaire ne sont donc pas nécessairement cloisonnés, mais peuvent s'interpénétrer, en particulier sur le plan sonore et, parfois, sur le plan musical. L'exemple de la musique de sc_e8ne, sur laquelle la Dame du radiateur se livre à une chorégraphie hasardeuse, traduit une irruption de la réalité diégétique dans le monde méta-diégétique. À l'inverse, on observe plusieurs fois, au cours du film, l'apparition d'une musique clairement méta-diégétique au sein de la réalité diégétique (Henry se remémore, à son propre rythme, certains passages de son disque). Dans un autre registre (celui de la comédie musicale), on peut mentionner Dancer in the Dark (Lars von Trier, 2000) qui instaure une double continuité entre bruits et musique, d'une part, 
entre monde réel et monde imaginaire, d'autre part. Mais ceci fera peut-être l'objet d'une autre étude.

\section{BIBLIOGRAPHIE}

CHION, Michel (2001). David Lynch. Paris : Cahiers du cinéma (Auteurs). 288 p.

CHION, Michel (1994). Le son au cinéma. Paris : Cahiers du cinéma (Essais). 220 p.

CHION, Michel (2003). Un art sonore, le cinéma : histoire, esthétique, poétique. Paris : Cahiers du cinéma (Essais). $478 \mathrm{p}$.

MILICEVIC, Mladen (nd). « Film Sound Beyond Reality: Subjective Sound in Narrative Cinema ». In FilmSound.org .[Internet]. Consulté en juillet 2008.

BRANIGAN, Edward (1984). Point of View in the Cinema: A Theory of Narration and Subjectivity in Classical Film. New York, Berlin : Mouton. 246 p.

GENETTE, Gérard (1972). Figures III. Paris : Seuil (Poétique). 286 p.

GORBMAN, Claudia (1987). Unheard Melodies: Narrative Film Music. London: BFI Publishing; Bloomington, Indiana: Indiana University Press. 190 p.

KROHN, Bill (1994). «Eraserhead : créer des sons, entretien avec David Lynch ». In Cahiers du cinéma, $\mathrm{n}^{\circ}$ 482, juillet-août 1994, pp. 14-17.

STEINLEIN, Almut (2007). Une esthétique de l'authentique : les films de la Nouvelle Vague. Paris : L'Harmattan, (Champs visuels). 269 p.

\section{NOTES}

1. Steinlein, $2007: 28$.

2. Gorbman, $1987: 23$.

3. Nous parlons, bien sûr, de focalisation interne, au sens où elle a été définie par Gérard Genette dans son analyse narratologique des modes du récit (Genette, 1972 : 203). Au cinéma, cette notion est étroitement liée à celles de « point de vue » (qui correspond, stricto sensu, à l'emplacement de la caméra) et de " point d'écoute ».

4. Au sein du modèle narratologique, l'application de la théorie de l'énonciation, formulée par Emile Benveniste, permet de déterminer le degré d'implication du narrateur fondamental dans le récit, ainsi que l'existence éventuelle d'un ou plusieurs narrateurs délégués - les "niveaux narratifs »-, autrement dit de répondre à la question «qui parle? » ou plus précisément «qui énonce? ». Comme les "récits emboîtés » de Genette, l'énonciation peut se situer à plusieurs niveaux ; ainsi, l'instance principale d'énonciation peut mettre en scène une instance se condaire qui, à son tour, produit un énoncé.

5. Branigan, 1984 : 94.

6. Chion, $2003: 429$.

7. Cf. le «tri-cercle » qui distingue le son in, le son hors-champ et le son off (Chion, 1994 : 33). 
8. Le cri d'une mouette dans un paysage de bord de mer sera interprété sans difficulté comme un son hors-champ ; en revanche, s'il jaillit en plein désert ou dans un vaisseau spatial, le spectateur est en droit de douter de la nature diégétique réelle de ce son.

9. Michel Chion cite le procédé utilisé dans Un grand amour de Beethoven (Abel Gance, 1937) afin d'illustrer la surdité du compositeur: le réalisateur «nous fai[t] d'abord entendre à nous, spectateurs, les sons du moulin, de l'oiseau et du violon, en nous faisant ensuite « basculer » dans le point d'écoute du malheureux qui ne les entend plus... ». Il mentionne également « la fameuse scène de gueule de bois dans le film de Jerry Lewis Docteur Jerry et Mister Love, 1963, lorsqu'au lendemain d'une de ses expériences de transformation, le malheureux professeur souffre de tous les bruits qu'il entend dans sa classe de chimie : la craie au tableau, le chewing-gum mâchouillé par une élève, lui deviennent des sensations énormes et insupportables - comme nous-mêmes, spectateurs, les entendons, démesurément grossies. » (Chion, $2003: 266$ ).

10. Dans Mulholland Drive (David Lynch, 2001), lorsque Dan se dirige vers l'arrière-cour du Winkie's pour aller à la rencontre de l'homme qui hante ses rêves, les bruits de la circulation nous parviennent de manière étouffée, assourdie ; quand il tombe au sol, terrassé par une crise cardiaque après avoir vu la figure monstrueuse, la voix de son ami résonne dans un écho lointain. 11. Plus rarement utilisé que le son remémoré, le son anticipé est notamment présent dans les films comportant des scènes de "visions" extra-lucides, par exemple The Dead Zone (David Cronenberg, 1984).

12. Chion, $2001: 54-55$.

13. Krohn, 1994 : 14.

\section{RÉSUMÉS}

Par définition, un film de fiction dépeint un monde imaginaire dans lequel évoluent - le plus souvent - des personnages, que le spectateur feint de considérer comme des êtres de chair et de sang, en vertu de ce que l'on nomme le " pacte de fiction ». Au sein de ce monde imaginaire ou monde diégétique, comment le spectateur peut-il faire la distinction entre ce qui est « réel », du point de vue des personnages, et ce qui ne l'est pas? En effet, contrairement à ce qui se produit dans la vie réelle, ce dernier peut, le cas échéant, avoir un accès direct, par le biais de l'image et/ ou du son, aux pensées, rêves et fantasmes des protagonistes. En nous appuyant sur la théorie de l'énonciation, adaptée au récit filmique, nous étudierons le rôle de la bande son dans la perception des mo ndes enchâssés, avant de proposer une classification des éléments sonores et musicaux (sons réels, sons subjectifs, sons imaginaires, etc.). Ce classement, qui repose à la fois sur la localisation de la source sonore et sur la détermination du point d'écoute, permet de rendre compte, sur le plan sonore, des procédés utilisés afin d'introduire une subjectivisation du récit filmique.

Nous illustrerons notre propos par une étude du traitement sonore dans le premier long métrage de David Lynch : Eraserhead. Ce film met en présence des espaces diégétiques réels et imaginaires qui, bien qu'ils soient clairement distincts, ne sont pas pour autant hermétiquement clos. L'interpénétration des espaces fictionnels devra mobiliser toute notre attention, puisque c'est précisément là, à la croisée des mondes (réel et imaginaire), que le traitement sonore joue un rôle prépondérant dans la compréhension du film. 


\section{AUTEUR}

\section{EMMANUELLE BOBÉE}

Doctorante en musicologie, Université de Rouen,

Assistante d'enseignement artistique en Conservatoire 